\title{
Spectroscopic Binary Candidates for Interferometers
}

\author{
Francis C. Fekel ${ }^{1}$ and Jocelyn Tomkin ${ }^{2}$ \\ ${ }^{1}$ Center of Excellence in Information Systems, Tennessee State University, \\ Nashville, TN 37209, USA \\ email: fekel@evans.tsuniv.edu \\ ${ }^{2}$ Department of Astronomy, University of Texas, Austin, TX 78712, USA \\ email: jt@alexis.as.utexas.edu
}

\begin{abstract}
We present a progress report on a program to improve the orbits of known spectroscopic binaries that are potential targets for ground-based optical interferometers, such as the CHARA array and PTI. The combination of such observations results in three-dimensional orbits that produce very accurate masses (uncertainties of less than $0.5 \%$ ) as well as orbital parallaxes that are generally more precise than those from Hipparcos. After additional analyses determine other basic parameters of the stars, the components can be compared with theoretical evolutionary tracks. We are currently using three observatories to obtain high-resolution, red-wavelength spectra of 44 systems. Results from four years of observation are highlighted. Interferometric observations of some systems are in progress.
\end{abstract}

Keywords. binaries: spectroscopic, stars: fundamental parameters

\section{Introduction}

With routine operation of several ground-based interferometers such as the Palomar Testbed Interferometer (PTI) (Colavita et al. 1999), the Naval Prototype Optical Interferometer (NPOI) (Hummel et al. 2003), and the Center for High Angular Resolution in Astronomy (CHARA) array (ten Brummelaar et al. 2003), the period overlap of spectroscopic and visual binaries has been greatly increased. The interferometric observations, combined with precise radial velocities, provide an opportunity to determine very accurate masses plus orbital parallaxes more accurate than those from Hipparcos for a significant number of systems.

\section{Binary sample}

We searched The Eighth Catalogue of the Orbital Elements of Spectroscopic Binary Systems (SB8) (Batten et al. 1989) for double-lined binaries that might be resolved interferometrically and could profit significantly from spectroscopic observations with modern CCD detectors. The new velocities would produce more precise minimum masses as well as updated orbital ephemerides to facilitate interferometric observations at the most appropriate orbital phases. Our initial list included 83 double-lined binaries north of $-30^{\circ}$ in declination, and generally brighter than $V=7.5 \mathrm{mag}$. In the hope of turning useless single-lined systems into valuable double-lined ones (e.g., Stockton \& Fekel 1992), we added to our list 50 single-lined binaries with mass functions greater than $0.05 M_{\odot}$. Also included were a few binaries from the more recent literature and systems with a giant component that we had previously been observing.

Of the 44 systems listed in Table 1, we have acquired at least 10 spectra for 37 . In addition to the HD number, we give the $V$ mag, spectral type of the primary, the 
Table 1. Observing list

\begin{tabular}{lclrrlllllrl}
\hline & $\begin{array}{c}V \\
\text { HD }\end{array}$ & $\begin{array}{l}\text { Spectral } \\
(\mathrm{mag})\end{array}$ & $\begin{array}{r}P \text { Type } \\
\text { (days) }\end{array}$ & $\begin{array}{c}\text { Mass } \\
\text { Ratio }\end{array}$ & $\begin{array}{l}\text { Pri- } \\
\text { ority }\end{array}$ & HD & $\begin{array}{c}V \\
(\mathrm{mag})\end{array}$ & $\begin{array}{l}\text { Spectral } \\
\text { Type }\end{array}$ & $\begin{array}{r}P \\
\text { (days) }\end{array}$ & $\begin{array}{r}\text { Mass } \\
\text { Ratio }\end{array}$ & $\begin{array}{c}\text { Pri- } \\
\text { ority }\end{array}$ \\
\hline 434 & 6.5 & Am & 34.3 & 0.84 & 2 & 120064 & 6.0 & F6IV & 36.0 & 0.85 & 2 \\
8374 & 5.6 & F1m & 35.4 & 0.98 & 2 & 141458 & 6.8 & A0V & 28.9 & 0.91 & 2 \\
9021 & 5.8 & F6V & 134.1 & 0.75 & 1 & 148367 & 4.6 & A5Vm & 27.2 & 0.85 & 3 \\
9312 & 6.8 & G5IV & 36.6 & 0.75 & 1 & 157950 & 4.5 & F2 & 26.3 & 0.63 & 1 \\
15138 & 6.1 & F4V & 11.0 & 0.93 & 2 & 160922 & 4.8 & F5V & 5.3 & 0.82 & 1 \\
20210 & 6.2 & F0m & 5.5 & 0.61 & 1 & 168913 & 5.6 & F0m & 5.5 & 0.85 & 2 \\
24623 & 7.1 & F2 & 19.7 & 0.98 & 3 & 170153 & 3.6 & F7Vmw & 280.6 & 0.72 & 1 \\
30453 & 5.9 & F0m & 7.0 & 0.66 & 1 & 171653 & 6.6 & Am & 14.3 & 0.94 & 2 \\
40084 & 5.9 & G5III & 219.1 & 0.98 & 1 & 171978 & 5.8 & A2V & 14.7 & 0.98 & 3 \\
42083 & 6.2 & A5III & 106.0 & 0.96 & 1 & 178619 & 6.5 & F5IV-V & 4.8 & 1.00 & 3 \\
61859 & 6.0 & F7V & 31.5 & 0.89 & 2 & 182490 & 6.2 & A1III & 7.4 & 0.74 & 1 \\
82191 & 6.6 & Am & 9.0 & 0.85 & 3 & 185734 & 4.7 & G8III & 434.1 & 0.96 & 2 \\
86146 & 5.1 & F5V & 9.3 & 0.80 & 1 & 188088 & 6.2 & K3V & 46.8 & 1.00 & 1 \\
93903 & 5.8 & Am & 6.2 & 0.50 & 1 & 191747 & 5.5 & A2IV & 9.3 & 0.91 & 2 \\
96511 & 7.1 & G0IV & 18.9 & 0.92 & 2 & 203439 & 6.0 & A1IV & 20.3 & 0.58 & 1 \\
102713 & 5.7 & F5IV & 32.9 & 0.80 & 1 & 205539 & 6.3 & F2IV & 12.2 & 0.88 & 2 \\
103578 & 5.5 & A3IV & 6.6 & 0.6 & 2 & 210027 & 3.8 & F5V & 10.2 & 0.62 & 2 \\
106677 & 6.4 & K0III & 64.4 & 0.98 & 1 & 210763 & 6.4 & F6V & 42.4 & 0.81 & 2 \\
108642 & 6.5 & A2m & 11.8 & 0.5 & 2 & 214686 & 6.9 & F7V & 21.7 & 0.99 & 3 \\
110318 & 5.2 & F5V & 44.4 & 0.50 & 1 & 218527 & 5.4 & G8III & 920.1 & 0.99 & 1 \\
112486 & 5.8 & A8m & 5.1 & 0.94 & 3 & 221950 & 5.7 & F6V & 45.5 & 0.94 & 3 \\
120005 & 6.6 & F5 & 39.3 & 0.58 & 1 & 224355 & 5.6 & F6V & 12.2 & 0.99 & 3 \\
\hline
\end{tabular}

orbital period, the secondary to primary mass ratio (mostly from our own work), and our observational priority. The primaries have spectral classes from early-A to early-K and are mostly dwarfs, but some subgiants and giants are included. The periods range from 5 to 920 days. Highest priority, a value of 1, has been assigned to those binaries with mass ratios significantly less than unity and also to systems containing evolved components. Such systems will have the greatest leverage in a comparison of observational results with theoretical evolutionary tracks.

\section{Spectroscopic observations}

Extensive observing began in 2002 April, and we are currently obtaining spectrograms at three observatories. Our observations at McDonald Observatory are acquired with the $2.1 \mathrm{~m}$ telescope and the Sandiford Cassegrain echelle spectograph. Those spectra cover the wavelength range $5600-7000 \AA$ and have a resolving power of 60,000 . Additional spectroscopic observations are collected with the Kitt Peak National Observatory $0.9 \mathrm{~m}$ coudé feed telescope, coudé spectrograph, and a TI CCD. The observations are centered at $6430 \AA$, cover a wavelength range of about $80 \AA$, and have a resolution of over 30,000 . Typical signal-to-noise ratios of the spectra are 250. Spectra of some binaries are being obtained at Fairborn Observatory with the $2 \mathrm{~m}$ automatic spectroscopic telescope and a fiber-fed echelle spectrograph with a SITe CCD. The wavelength range is $4920-7100 \AA$, and the resolving power is about 35,000 .

\section{Results}

We have detected the secondaries of 10 binaries with previous single-lined orbits, HD 434, HD 9021, HD 9312, HD 20210 (Fig. 1), HD 96511, HD 102713, HD 103578, HD 


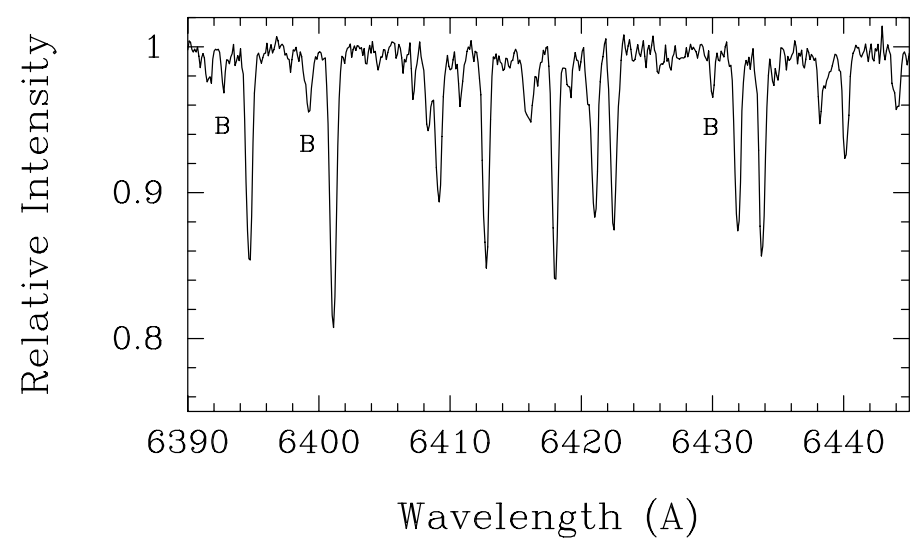

Figure 1. Spectrum of HD 20210. Several lines of component B are indicated.

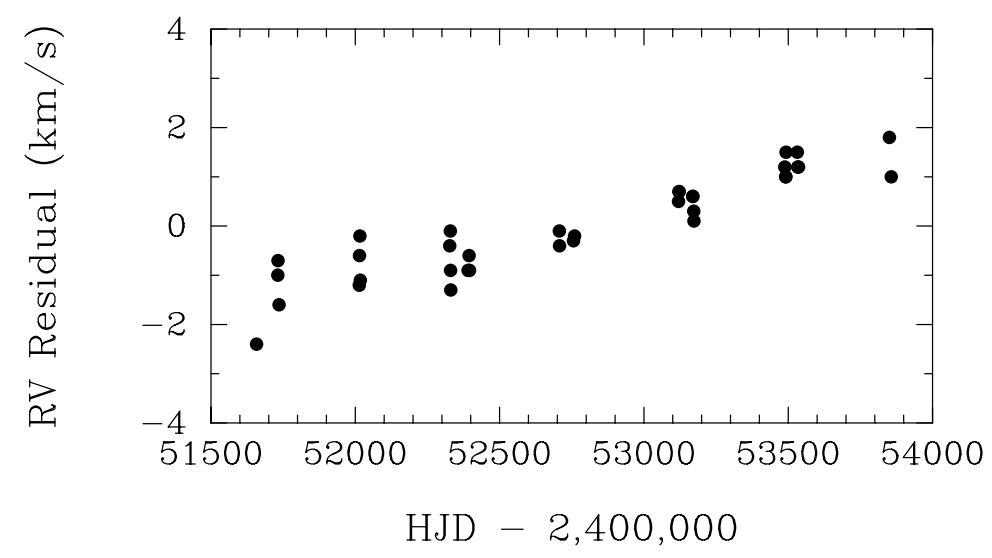

Figure 2. Velocity residuals from a circular orbit fit of HD 108642A.

108642, HD 110318, and HD 120005. Velocities for two of those systems, HD 103578 and HD 108642 (Fig. 2), have systematic residuals, so the systems are at least triple. Our spectra of HD 148367 have three sets of lines, so that system also is at least triple. Tomkin \& Fekel (2006) published the first results of our program, new spectroscopic orbits for RR Lyn, 12 Boo, and HR 6169. Because RR Lyn is eclipsing and 12 Boo already has an interferometric orbit, we obtained masses with a precision better than $0.5 \%$ for the components. Our new orbit of HR 6169 produced significantly revised minimum masses. Many of our top priority systems are now being observed with the CHARA array.

\section{Acknowledgements}

This work is supported by NSF grant HRD-9706268 to Tennessee State University.

\section{References}

Batten, A.H., Fletcher, J.M., \& MacCarthy, D.G. 1989, Publ. Dom. Astrophys. Obs. 17, 1

Colavita, M.M., et al. 1999, ApJ 510, 505

Hummel et al. 2003, AJ 125, 2630

Stockton, R.A., \& Fekel, F.C. 1992, MNRAS 256, 575

ten Brummelaar, T.A., et al. 2003, Proc. SPIE 4838, 69

Tomkin, J., \& Fekel, F.C. 2006, AJ 131, 2652 